\title{
Effectiveness evaluation of FTN signaling in the presence of nonlinear distortion under multipath fading channels
}

\author{
Atsuya Nakamura ${ }^{1}$, Yuta Kumagai ${ }^{1}$, Shuhei Saito ${ }^{1}$, \\ Hirofumi Suganuma ${ }^{1}$, Keita Kuriyama ${ }^{2}$, Yu Ono ${ }^{2}$, \\ Hayato Fukuzono $^{2}$, Masafumi Yoshioka ${ }^{2}$, \\ and Fumiaki Maehara ${ }^{1 \mathrm{a})}$ \\ ${ }^{1}$ Graduate School of Fundamental Science and Engineering, Waseda University, \\ 3-4-1 Ohkubo, Shinjuku-ku, Tokyo 169-8555, Japan \\ ${ }^{2}$ NTT Access Network Service Systems Laboratories, Nippon Telegraph and \\ Telephone Corporation, \\ 1-1 Hikarino-oka, Yokosuka-shi, Kanagawa 239-0847, Japan \\ a)fumiaki_m@waseda.jp
}

\begin{abstract}
This study investigates the throughput performance of fasterthan-Nyquist (FTN) signaling in the presence of nonlinear distortion under multipath fading channels. In our performance evaluation, we use a practical transmission method, in which single-carrier with frequency-domain equalization (SC-FDE) is applied to FTN signaling for broadband wireless communications. For a radio propagation model, the 3GPP tapped delay line (TDL) model is used to evaluate the effect of multipath fading, and the Rapp model is adopted as a power amplifier (PA) model that provides the impact of nonlinear distortion. We compare the throughput performance of FTN signaling with that of Nyquist signaling under the same transmission rate via computer simulations, which clarifies the applicability of FTN signaling to practical broadband wireless communications.
\end{abstract}

Keywords: Faster-than-Nyquist (FTN) signaling, nonlinear distortion, multipath fading, peak-to-average power ratio (PAPR), throughput.

Classification: Wireless Communication Technologies

References

[1] Cisco, "Cisco annual internet report (2018-2023)," White Paper, Mar. 2020.

[2] J. E. Mazo, "Faster-than-Nyquist signaling," Bell Syst. Tech. J., vol. 54, no. 8, pp. 1451-1462, Oct. 1975.

[3] J. Fan, S. Guo, X. Zhou, Y. Ren, G. Y. Li, and X. Chen, "Faster-than-Nyquist signaling: An Overview," IEEE Access, vol. 5, pp. 1925-1940, Feb. 2017.

[4] T. Ishihara, S. Sugiura, and L. Hanzo, "The evolution of faster-than-Nyquist signaling," IEEE Access, vol. 9, pp. 86535-86564, June 2021.

[5] J.-A. Lucciardi, N. Thomas, M.-L. Boucheret, C. Poulliat, and G. Mesnager, "Trade-off between spectral efficiency increase and PAPR reduction when using FTN signaling: Impact of non linearities," Proc. 2016 IEEE Int. Conf. Commun. (ICC 2016), pp. 1-7, May 2016.

[6] 3GPP TR38.901 v14.2.0, "Study on channel model for frequencies from 0.5 to $100 \mathrm{GHz},{ }^{\prime}$ Sept. 2017. 
[7] C. Rapp, "Effects of HPA-nonlinearity on a 4-DPSK/OFDM-signal for a digital sound broadcasting system," Proc. 2nd Eur. Conf. Satell. Commun., pp. 179184, Oct. 1991.

[8] L. Cariou and G. Venkatesan, “TGay evaluation methodology," IEEE 802.1115/0866r2, Jan. 2016.

[9] J. Zeng, Y. Wang, X. Zhang, K. Hu, and Y. Wang, "Reduced-complexity IBDFE for faster-than-Nyquist communication systems," Proc. 2016 IEEE Mil. Commun. Conf. (MILCOM 2016), pp. 654-659, Nov. 2016.

[10] K. Kojima, Y. Shimbo, H. Suganuma, and F. Maehara, "Deep learning based CoMP transmission method using vehicle position information for taxi radio systems," Proc. 2020 Int. Conf. Artificial Intell. Inf. Commun. (ICAIIC 2020), pp. 253-256, Feb. 2020.

\section{Introduction}

The diversification of mobile network services has led to an increase in the number of wireless devices, and hence, the growth of mobile traffic. The number of global mobile connections is expected to increase to 13.1 billion by 2023 [1]. Faster-than-Nyquist (FTN) signaling is a promising technique for improving spectral efficiency $[2,3,4]$. This is because FTN signaling performs non-orthogonal transmission that compresses the symbol period in the time domain while allowing inter-symbol interference (ISI), thereby increasing the transmission rate compared with Nyquist signaling. Thus, FTN signaling is considered suitable for application to systems such as mobile communications systems and broadcasting satellite systems [3].

In FTN signaling, because the induced ISI affects the peak-to-average power ratio (PAPR) of the transmitted signal, the performance evaluation considering the nonlinear distortion caused by the power amplifier (PA) is meaningful and important. In [5], a performance comparison between FTN signaling and traditional Nyquist signaling for DVB-S2 under a constant transmission rate was provided. This study showed that FTN signaling has great potential to improve the system capacity while retaining a low PAPR because of the use of lower modulation levels owing to the compressed symbol period. However, because this study assumed only an additive white Gaussian noise (AWGN) channel, a performance evaluation under multipath fading channels is expected considering its applicability to broadband wireless communications. In particular, because FTN signaling essentially requires equalization at the receiver, its performance is strongly affected by both ISI and multipath fading.

Considering the background described above, we investigate the throughput performance of FTN signaling in the presence of nonlinear distortion under multipath fading channels. Single-carrier with frequency-domain equalization (SC-FDE) is applied to FTN signaling as a practical transmission method for broadband wireless communications [4]. As a radio propagation model, the 3GPP tapped delay line (TDL) model is considered [6], which enables us to consider the applicability of FTN signaling to millimeter-wave (mmWave) bands below $100 \mathrm{GHz}$. For the PA model, the Rapp model is adopted [7, 8], which makes it possible to ensure the 
impact of nonlinear distortion on FTN signaling. The performance comparisons between FTN signaling and Nyquist signaling are demonstrated by means of computer simulations, which clarifies the applicability of FTN signaling to broadband wireless communications under multipath fading channels.

\section{System model}

In this section, we briefly introduce the operating principles of FTN signaling. Fig. 2 illustrates the system configuration of FTN signaling employing SC-FDE. At the transmitter, the modulated signal with an $N_{G}$-length guard interval (GI) $a[n]$ is pulse-shaped by a low-pass filter (LPF) $g(t)$. The pulse-shaped signal $s(t)$ is given by

$$
s(t)=\sum_{n} a[n] g(t-n \tau T),
$$

where $T$ denotes the symbol period under the Nyquist criterion, and $\tau(0<\tau \leq 1)$ is the compression factor. It should be noted that the transmission rate of FTN signaling can be increased up to $1 / \tau$ times that of Nyquist signaling $(\tau=1)$. The pulse-shaped signal is enhanced by a power amplifier (PA) and then transmitted.

At the receiver, the received signal $r(t)$ is passed through the matched filter $g^{*}(-t)$ and then sampled every time $\tau T$. In multipath fading channels, which should be considered, especially in mobile broadband communications, the received signal after the matched filter $y[n]$ is represented by

$$
\begin{aligned}
y[n] & =\int_{-\infty}^{\infty} r(t) g^{*}(-(t-n \tau T)) d t \\
& =\sum_{l=0}^{L-1} \sum_{m=-v}^{+v} a[m] h[l] q[n-(l+m)]+\eta[n],
\end{aligned}
$$

where $h[l]$ is the channel impulse response, $L$ is its effective length, $(2 v+1)$ corresponds to the tap length consisting of the transmitting and receiving filters, and $q[n-m]$ and $\eta[n]$ are defined as

$$
\begin{aligned}
q[n-m] & =\int_{-\infty}^{\infty} g(t-m \tau T) g^{*}(-(t-n \tau T)) d t, \\
\eta[n] & =\int_{-\infty}^{\infty} n(t) g^{*}(-(t-n \tau T)) d t .
\end{aligned}
$$

Here, $n(t)$ is the white Gaussian noise with power spectral density $N_{0}$. By using Eq. (2), the received signal vector $\mathbf{y}$ for each $N_{F}$-length block can be expressed as follows:

$$
\mathbf{y}=\mathbf{H Q} \mathbf{a}+\mathbf{n},
$$

where $\mathbf{a}=\left[a[0], a[1], \cdots, a\left[N_{F}-1\right]\right]^{\mathrm{T}} \in \mathbb{C}^{N_{F}}$ and $\mathbf{n}=\left[\eta[0], \eta[1], \cdots, \eta\left[N_{F}-1\right]\right]^{\mathrm{T}} \in$ $\mathbb{C}^{N_{F}}$ are the modulated signal and colored noise vectors, respectively; $\mathbf{H} \in \mathbb{C}^{N_{F} \times N_{F}}$ and $\mathbf{Q} \in \mathbb{C}^{N_{F} \times N_{F}}$ are circulant matrices populated with tap coefficients $h[\cdot]$ and $q[\cdot]$, respectively. The time-domain received signal vector $\mathbf{y}$ is transformed into the frequency-domain signal $\mathbf{y}_{f}$, which is represented by

$$
\begin{aligned}
\mathbf{y}_{f} & =\mathbf{F}(\mathbf{H Q a}+\mathbf{n}) \\
& =\mathbf{\Lambda} \mathbf{a}_{f}+\mathbf{n}_{f},
\end{aligned}
$$




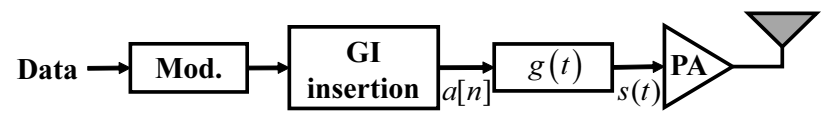

(a) Transmitter

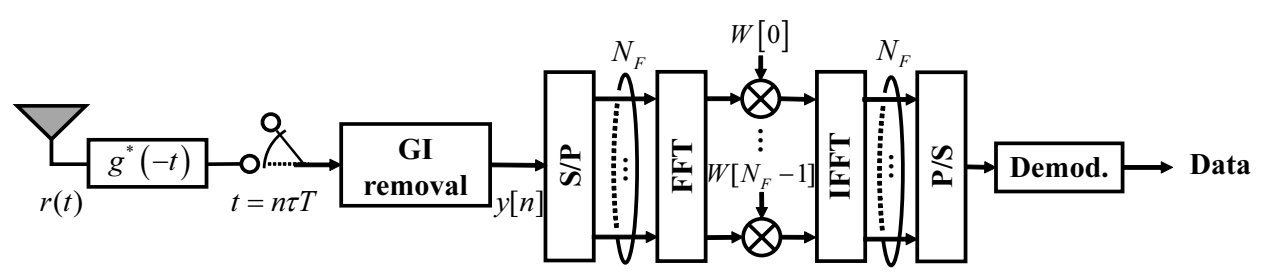

(b) Receiver

Fig. 1. System configuration of FTN signaling employing SC-FDE.

where $\mathbf{F} \in \mathbb{C}^{N_{F} \times N_{F}}$ denotes the $N_{F}$-point fast Fourier transform (FFT) matrix, and the circulant matrix $\mathbf{H Q}$ can be diagonalized by $\mathbf{H Q}=\mathbf{F}^{\mathrm{H}} \boldsymbol{\Lambda} \mathbf{F}$. $\mathbf{a}_{f} \in \mathbb{C}^{N_{F}}$ and $\mathbf{n}_{f} \in$ $\mathbb{C}^{N_{F}}$ are the frequency-domain modulated signal and noise vectors, respectively. Here, the $(k, l)$ element of $\mathbf{F}$ is given by

$$
[\mathbf{F}]_{k, l}=\frac{1}{\sqrt{N_{F}}} \exp \left(-j \frac{2 \pi k l}{N_{F}}\right) .
$$

Assuming the minimum mean square error (MMSE) as the equalization criterion, the FDE weight matrix $\mathbf{W} \in \mathbb{C}^{N_{F} \times N_{F}}$ is given by [4]

$$
\begin{aligned}
\mathbf{W} & =\boldsymbol{\Lambda}^{\mathrm{H}}\left(\boldsymbol{\Lambda} \mathbf{\Lambda}^{\mathrm{H}}+\frac{1}{E_{s}} \mathbb{E}\left[\mathbf{n}_{f} \mathbf{n}_{f}^{\mathrm{H}}\right]\right)^{-1} \\
& \simeq \boldsymbol{\Lambda}^{\mathrm{H}}\left(\boldsymbol{\Lambda} \mathbf{\Lambda}^{\mathrm{H}}+\frac{1}{E_{s}} \boldsymbol{\Phi}\right)^{-1},
\end{aligned}
$$

where $\boldsymbol{\Phi}=\operatorname{diag}\left(\phi[0], \phi[1], \cdots, \phi\left[N_{F}-1\right]\right) \in \mathbb{C}^{N_{F} \times N_{F}}$ is a diagonal matrix whose elements are calculated by

$$
\begin{aligned}
\phi[k] & =\mathbb{E}\left[\frac{1}{N_{F}}\left|\sum_{m=0}^{N_{F}-1} \eta[m] e^{-j \frac{2 \pi k m}{N_{F}}}\right|^{2}\right] \\
& =\frac{N_{0}}{N_{F}} \sum_{n=0}^{N_{F}-1} \sum_{m=0}^{N_{F}-1} q[n-m] e^{-j \frac{2 \pi(n-m) k}{N_{F}}} .
\end{aligned}
$$

Using the diagonal weight matrix $\mathbf{W} \simeq \operatorname{diag}\left(W[0], W[1], \cdots, W\left[N_{F}-1\right]\right)$ of Eq. (8), the time-domain signal after FDE $\hat{\mathbf{a}} \in \mathbb{C}^{N_{F}}$ is obtained as follows:

$$
\hat{\mathbf{a}}=\mathbf{F}^{\mathrm{H}} \mathbf{W F y} \text {. }
$$

\section{Numerical results}

In this section, we present a performance comparison between FTN and Nyquist signaling in the presence of nonlinear distortion under multipath fading channels by means of computer simulations. Table $\mathbf{I}$ lists the simulation parameters. In our performance evaluation, a 4-bit transmission was assumed. Specifically, the 8PSK 
Table I. Simulation parameters

\begin{tabular}{c|c}
\hline Carrier frequency $f_{c}$ & $70 \mathrm{GHz}$ \\
\hline Bandwidth $B$ & $1.0 \mathrm{GHz}$ \\
\hline Sampling period $T_{\text {sam }}$ & $0.375 \mathrm{~ns}$ \\
\hline Modulation & 8PSK (FTN, $\tau=0.75$ ), 16QAM (Nyquist), \\
& 16APSK (Nyquist, Radius ratio = 2.57) \\
\hline Number of FFT points $N_{F}$ & 2048 \\
\hline GI length $N_{G}$ & 512 \\
\hline Pulse shaping filter & Root-raised cosine (RRC) \\
\hline Roll-off factor $\alpha$ & 0.5 \\
\hline Channel model & 3GPP TDL-D [6] \\
\hline Delay spread $\tau_{r m s}$ & $26 \mathrm{~ns}$ \\
\hline Channel estimation & Perfect \\
\hline
\end{tabular}

modulation by FTN signaling with $\tau=0.75$ was compared with the 16APSK and 16QAM modulations by Nyquist signaling. Moreover, a root-raised-cosine (RRC) filter with a roll-off factor of $\alpha=0.5$ was used as a pulse-shaping filter [9]. At the transmitter, the Rapp model was adopted for a PA, and its input-output relationship was given by $[7,8]$

$$
A_{\text {out }}(t)=\frac{G_{0} A_{\text {in }}(t)}{\left[1+\left(\frac{A_{\text {in }}(t)}{A_{\text {sat }}}\right)^{2 \rho}\right]^{\frac{1}{2 \rho}}},
$$

where $G_{0}$ is the power gain, $A_{\text {in }}(t)$ and $A_{\text {out }}(t)$ are the input and output signal amplitudes, respectively, and $A_{s a t}$ is the saturation input amplitude. Herein, the nonlinear factor $\rho$ and input back-off (IBO) $\zeta$ were set to 0.81 and $0.5 \mathrm{~dB}$, respectively [8]. Furthermore, considering that the propagation environment is mainly at line-of-sight (LOS) in mmWave broadband communications, the 3GPP TDL-D model [6] was assumed as the radio propagation model, where the delay spread $\tau_{r m s}$ was set to $26 \mathrm{~ns}$ in the carrier frequency $f_{c}=70 \mathrm{GHz}$. It was noted that the GI length $N_{G}$ was longer than the filter and channel delay; therefore, there was no effect of the inter-block interference (IBI).

Fig. 2(a) shows the complementary cumulative distribution function (CCDF) of the PAPR. From Fig. 2(a), it is observed that the PAPR of FTN signaling with 8PSK is significantly smaller than that of Nyquist signaling with 16QAM. This is because by compressing the symbol period, FTN signaling can reduce the modulation level while retaining the transmission rate. Moreover, the FTN signaling with 8PSK achieves almost the same PAPR as Nyquist signaling with 16APSK, characterized by a relatively small number of possible amplitude levels.

Fig. 2(b) shows the throughput performance versus the average carrier-to-noise ratio $(\mathrm{CNR}) \Gamma$. Here, the throughput is calculated by $[4,10]$

$$
\text { Throughput }=\frac{N_{F}}{N_{F}+N_{G}} \cdot \frac{\log _{2} M}{\tau(1+\alpha)} \cdot\left(1-H\left(P_{e}\right)\right),
$$

where $M$ is the modulation level, $H(\cdot)$ is the binary entropy function, and $P_{e}$ is the uncoded bit error rate obtained by computer simulations. In the absence of nonlinear distortion $(\zeta=\infty)$, as shown in Fig. 2(b)-(i), the throughput of FTN signaling is better than that of Nyquist signaling when $\Gamma<10 \mathrm{~dB}$. This is because the reduction of the modulation level in FTN signaling leads to improved robustness 


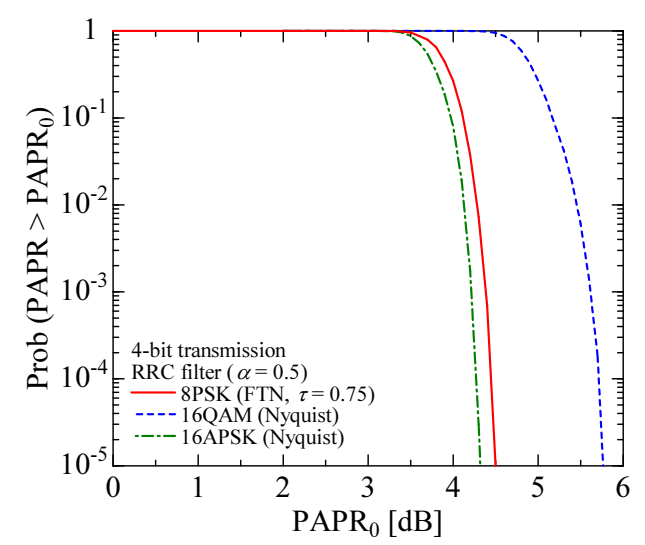

(a) CCDF of PAPR

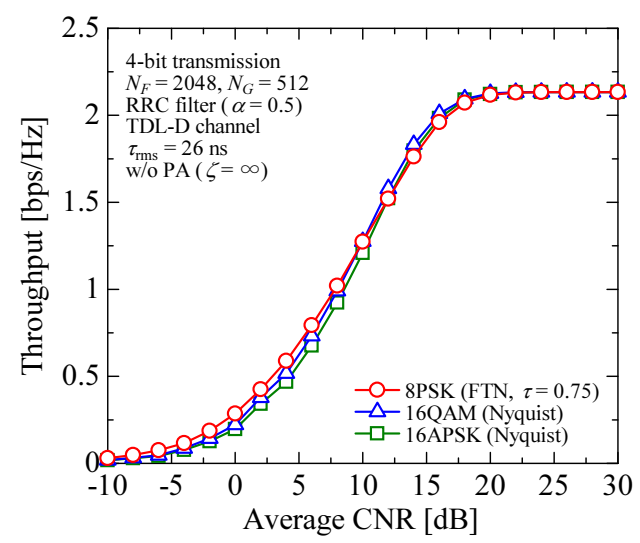

(i) w/o nonlinear PA

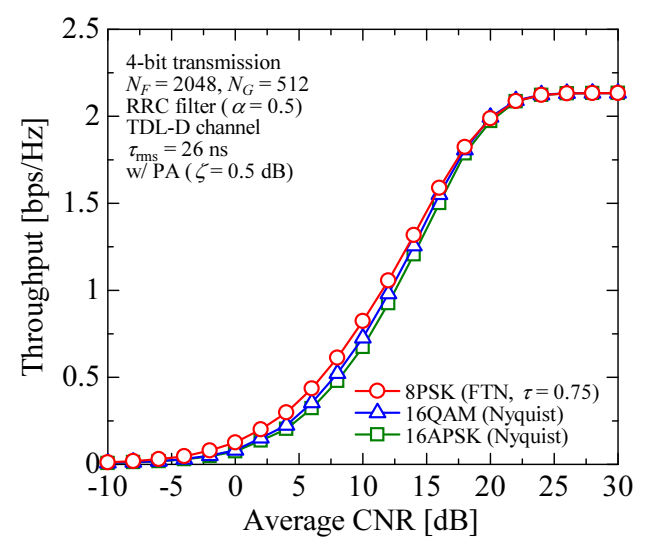

(ii) $\mathrm{w} /$ nonlinear PA

(b) Throughput versus average CNR

Fig. 2. Performance comparison between FTN signaling and Nyquist signaling.

against noise. In the presence of nonlinear distortion $(\zeta=0.5 \mathrm{~dB})$, as shown in Fig. 2(b)-(ii), the CNR region where FTN signaling outperforms Nyquist signaling is enlarged. In particular, the difference between FTN signaling and Nyquist signaling with 16QAM increases. This is because FTN signaling with 8PSK has a lower PAPR, which alleviates the impact of nonlinear distortion.

\section{Conclusion}

In this study, we investigated the throughput performance of FTN signaling by employing SC-FDE in the presence of nonlinear distortion under multipath fading channels. In our performance evaluation, the effect of frequency-selective fading channels, as well as nonlinear distortion, which should be considered for broadband wireless communications, were incorporated. The numerical results showed that the PAPR of FTN signaling with 8PSK becomes dramatically smaller than that of Nyquist signaling with 16QAM because FTN signaling can reduce the modulation level while retaining the transmission rate. Moreover, the throughput of FTN signaling is better than that of Nyquist signaling, which indicates that the use of a lower modulation level with receiver equalization is robust against multipath fading as well as nonlinear distortion. 